\author{
Radmila Lazarevic ${ }^{*}$ \\ Università del Montenegro
}

\title{
FALSI AMICI DI TUTTI I COLORI: \\ I TRANELLI SEMANTICI NELLA TRADUZIONE DELLE ESPRESSIONI CROMATICHE ITALIANE E SERBE
}

Abstract: Il contributo tratta le espressioni polirematiche italiane e serbe che includono aggettivi per i colori basici (in base alla nomenclatura di Berlin e Kay del 1969), sia i modi di dire sia le collocazioni fisse. In una lingua un certo modo di dire può ricoprire più significati diversi che nell'altra (morte bianca, nastro azzurro, žuto zlato), oppure i significati dei sintagmi in apparenza traducibili alla lettera possono differire completamente (peste bianca/bela kuga, valuta verde/zelena valuta). Gli esempi del genere, in cui la vera equivalenza semantica non esiste, sono riscontrati nelle sfere più svariate della vita, per cui è necessario controllare accuratamente i possibili significati delle polirematiche che a prima vista sembrano traduzione letterale. Questo articolo riporta alcuni esempi di cosiddetti "falsi amici" tra le espressioni e collocazioni cromatiche che potrebbero rappresentare difficoltà nella traduzione dall'italiano in serbo e viceversa.

Parole chiave: colori basici, falsi amici, italiano, semantica, serbo, traduzione

\section{INTRODUZIONE}

Negli ultimi decenni le ricerche sul campo della terminologia cromatica si sono sviluppate soprattutto seguendo la teoria proposta da Brent Berlin e Paul Kay nel 1969, secondo cui (dopo numerosi esperimenti che finora hanno incluso parlanti di oltre cento lingue del mondo) è possibile individuare una nomenclatura universale di undici colori basici, che sarebbero bianco, nero, rosso, verde, giallo, arancione, azzurro o blu, marrone, rosa, viola e grigio (Berlin e Kay 1999; Lazarević 2013a). Non tutte le lingue studiate annoverano ciascuno di questi termini basici, ma sono presenti nella maggioranza delle lingue europee, tra cui sono anche l'italiano e il serbo (nel serbo: bela, crna, crvena, zelena, žuta, narandžasta, plava, smeđa, ružičasta, ljubičasta e siva).

*radmilal@ac.me 
Da una estesa ricerca comparativa sulla terminologia cromatica nelle lingue italiana e serba ${ }^{1}$, che comprende vari valori semantici e simbolici dei suddetti aggettivi cromatici, nonché modi di dire e collocazioni che li includono, per questa occasione ci fermeremo sulle locuzioni o collocazioni cromatiche, cioè sui sintagmi o le espressioni polirematiche che includono uno dei colori basici come interpretati da Berlin e Kay.

\subsection{Il corpus lessicografico}

Il corpus per la ricerca consiste dei dizionari monolingui italiani e serbi (De Mauro 2000, Devoto-Oli 2006, Garzanti 2006, Sabatini-Coletti 2006, Zingarelli 2008 e il sito dell'Accademia Treccani www.treccani.it, poi Rečnik srpskohrvatskoga književnog jezika I-VI (1967-1976) e Rečnik srpskoga jezika (2007). Questi materiali sono stati integrati con due dizionari fraseologici, Frazeološki rječnik hrvatskog ili srpskog jezika di Josip Matešić (1982) e Frazeološki rečnik srpskog jezika di Đorđe Otašević (2012), allo scopo di completare il corpus serbo, che a tutt'oggi manca di varietà lessicografica riguardo al numero di edizioni diverse o aggiornate.

Visto che i dizionari serbi non presentano un numero notevole di collocazioni e modi di dire contenenti colori basici in uso quotidiano sia nel parlato sia nel linguaggio dei media (ad es. biti zaštićen kao beli medved, račun u crvenom, zelena energija ecc.), abbiamo creduto necessario di arricchire il corpus, soprattutto quello serbo, di un certo numero di espressioni tratte dalla stampa e da Internet, come esempi di quel linguaggio contemporaneo che non ha ancora trovato il proprio posto sulle pubblicazioni lessicografiche (Lazarević 2013a). Perciò sono stati inclusi pure gli esempi non riportati dal corpus lessicografico serbo (o serbocroato) che trovano i propri "falsi amici" o false analogie nelle collocazioni o locuzioni cromatiche italiane (siva faza, pobediti za zelenim stolom, zelena karta ecc.), ed anche alcune polirematiche che non si trovano sui dizionari italiani, seppure in numero trascurabile (green card, televisione rosa, economia grigia, sport bianco, ecc). Naturalmente, il numero totale di esempi rilevati è limitato, visto che anche i "falsi amici" tra le espressioni polirematiche sono relativamente pochi in confronto a quelli che si possono ricavare tra le monorematiche. Quindi, gli esempi qui riportati dovrebbero servire a scopo illustrativo, per evidenziare alcuni specifici tranelli da evitare nella traduzione.

${ }^{1}$ La ricerca del dottorato, per la tesi intitolata Il campo lessico-semantico dei colori nelle lingue italiana e serba. 


\subsection{La scelta del termine}

Il termine "falsi amici" qui è inteso soprattutto dal punto di vista semantico, nel senso più ampio contribuito a questo fenomeno da vari autori che si sono occupati della terminologia, e che hanno diviso questa categoria in falsi amici morfologici e in quelli semantici (Benedik e Gabrovšek 2004; Benincà e Penello 2006; Buntić 1990; Ivir 1968, 1984; Kudela 1981; Lewis 2008; Matešić 1995; Vuletić 2010). E stato scelto proprio il termine "falsi amici" come il più noto e adoperato sia nel serbo sia nell'italiano (dove troviamo anche dei sinonimi meno usati, come parole trappola, falsi affini, false analogie, ambigue affinità, tranelli di una lingua ecc.).

Non parleremo a questo proposito dell'omonimia interlinguistica, visto che il fenomeno in questione si può manifestare non soltanto nella somiglianza morfologica ed ortoepica tra le parole che rappresenta l'omonimia per definizione, ma anche negli errori di traduzione letterale, basati sul significato dei singoli componenti nelle espressioni polirematiche (Ivir 1984; Vuletić 2010).

Il contributo tratta soprattutto gli ultimi, falsi amici semantici o fraseologici (Lewis, 2008), benché tra gli esempi analizzati riscontriamo pure i casi di "classica" omonimia interlinguistica (carta verde/zelena karta, fase grigia/siva faza). Se tra queste espressioni sussiste un'omofonia, sarà legata di norma al componente non cromatico dell'espressione, che di solito è il sostantivo. Il principale errore da evitare nel tradurre questi sintagmi, come ci ammonisce Klajn, è ricorrere alla traduzione letterale soltanto perché a prima vista assomigliano a quelli presenti nella lingua di destinazione: "Leksički paralelizmi između dva jezika dovode do pojave 'lažnih prijatelja' (falsi amici), kako se popularno nazivaju reči analognog oblika a različitog značenja... Ponekada i prevod dužih izraza može da bude mehanički, gotovo automatski..." (1971: 32). Falsi amici fraseologici a volte finiscono col diventare calchi, e i calchi dalle altre lingue rappresentano una possibile fonte di malintesi (Klajn 1971); comunque, nel caso degli esempi riscontrati nel nostro corpus, non sempre risulta possibile parlare dei calchi, né dimostrare la provenienza esatta di una locuzione.

\section{FALSI AMICI SEMANTICI}

In una gran parte dei casi una sola delle due lingue possiede l'espressione cromatica con un determinato significato. In alcuni casi specifici, è possibile invece che l'espressione italiana ricopra un campo semantico più esteso rispetto alla sua controparte serba (o viceversa), ma può darsi anche che i significati delle espressioni nelle due lingue, benché equivalenti in apparenza, differiscano completamente (morte bianca/bela smrt, peste 
bianca/bela kuga, carta verde/zelena karta, ecc.). Anche il significato generico di un colore basico preso a sé stante può variare da una lingua all'altra, come anche il suo potenziale semantico, il che comporta una certa asimmetria tra i campi semantici nelle due lingue. Qui dedicheremo attenzione particolare ai casi in cui i termini italiani e serbi coincidono, ma i loro significati sono diversi.

Osservando le relazioni tra le espressioni polirematiche italiane e serbe che includono colori basici, $i$ falsi amici semantici ${ }^{2}$ possono essere suddivisi in:

a) quelli che ricoprono significati completamente diversi, che chiameremo falsi amici totali

b) quelli con più significati, tra cui uno può corrispondere alla sua controparte nell'altra lingua, mentre l'altro/gli altri significati ricoprono campi semantici differenti o anche opposti. Di solito questa polisemia non si manifesta che in una delle due lingue, mentre nell'altra la rispettiva espressione ha un solo significato che non corrisponde a quello della traduzione letterale. Questi sono i falsi amici parziali, che possono essere definiti anche polisemici (Kudela 1981).

Per quanto riguarda gli ambiti d'uso, le espressioni polirematiche equivalenti o meno si possono trovare nei settori più disparati: storia, geografia, medicina, biologia, sport, politica, economia... Le collocazioni o le locuzioni cromatiche che nell'italiano appartengono ad un determinato settore, come fisica o biologia, nel serbo si possono riferire a tutt'altro, ad esempio all'edilizia (cfr. fase grigia/siva faza). Perciò non verranno classificate secondo il criterio dei rispettivi settori, ma piuttosto secondo quello più formale del colore basico il cui campo semantico ricoprono.

\subsection{Falsi amici totali}

La particolare attenzione va dedicata ad alcune espressioni le cui somiglianze potrebbero portare alle traduzioni erronee. Qui sono riportati alcuni esempi classificati per colori basici.

\subsubsection{ROSSO}

Nei toponimi, il serbo adopera la perifrasi "crveno ostrvo" per Cuba; in italiano, però, Isola Rossa è nome di un luogo in Sardegna, oppure si tratta della perifrasi usata più per il Madagascar che non per Cuba.

${ }^{2}$ La denominazione di queste due categorie di falsi amici semantici è stata ripresa da Benincà e Penello (2006). 


\subsubsection{VERDE}

La locuzione italiana vincere al tavolo verde significa essere fortunati nei giochi d'azzardo, mentre il serbo pobediti za zelenim stolom nella terminologia sportiva vuol dire vincere una partita col risultato ufficiale, senza giocare. La locuzione italiana essere al verde (rimanere senza un soldo) differisce completamente dalla serba, apparentemente simile, doći na zelenu granu (cavarsi da una situazione difficile, riprendersi materialmente, arricchirsi; Lazarević 2013b). La perifrasi serba zelena valuta o zelena novčanica si riferisce al dollaro americano (in italiano biglietto verde), a differenza dell'italiana moneta/valuta verde che viene adoperata nel contesto dell'Unione europea, per i prezzi dei prodotti agricoli determinati da certi tassi di cambio valutario.

La carta verde italiana non corrisponde alla serba zelena karta, ma alla collocazione zeleni karton (il certificato internazionale di assicurazione per i veicoli), mentre zelena karta (calco dall'inglese green card) si riferisce al permesso di residenza negli USA a tempo illimitato per uno straniero, che l'italiano ha ripreso nella forma originale, come prestito diretto, e quindi per questo significato non viene adoperato il calco.

\subsubsection{ROSA}

La televisione rosa italiana (una televisione che tratta gli stessi argomenti della cronaca rosa, ad esempio) non equivale al serbo "ružičasta televizija", che ha il significato specifico associato alla TV Pink serba, che si è diffusa anche in altre regioni dei Balcani.

\subsubsection{GRIGIO}

Uno dei significati dell'aggettivo grigio si può avvicinare al significato dell'aggettivo nero quando indica un'attività illegale, clandestina, o un modo di concludere affari aggirando le norme giuridiche, cercandovi dei "buchi" (dal punto di vista semantico, si può associare alla "zona grigia" dove i confini etici tra giusto e sbagliato sono vaghi e confusi).

La collocazione serba siva faza di recente viene adoperata sempre più spesso, nell'ambito dell' edilizia e del mercato immobiliare, per definire la fine dei lavori in fase di esecuzione, dove la rifinitura dell'interiore di solito viene lasciata al cliente. Anche questo termine contiene una connotazione di incertezza e spesso viene collegato con le attività sull'orlo della legalità, essendo pertanto semanticamente affine ai termini come siva zona e siva ekonomija. La collocazione italiana fase grigia non è equivalente, né ha un significato univoco; calco dall'inglese grey phase, descrive la fase di transizione in un processo duraturo, e si può riferire a diversi settori (me- 
dicina, zoologia, psicologia, ecc.). L'elemento comune a questi contesti differenti è il fatto che nella maggior parte dei casi si tratta di un periodo difficile che va superato.

\subsubsection{GIALLO}

È tipico della lingua italiana l'uso dell'aggettivo giallo per nominare alcuni prodotti dall'Estremo Oriente. Così, il sintagma auto gialla di frequente si riferisce a una macchina prodotta, ad esempio, in Giappone, a differenza del serbo dove žuti automobil avrebbe il significato letterale, e quindi può facilmente essere frainteso.

\subsubsection{BIANCO}

Il sintagma italiano peste bianca si riferisce alla tubercolosi, e nei tempi recenti anche alla droga, mentre il serbo bela kuga definisce il tasso di natalità in calo, e quindi non rappresenta un equivalente traduttivo (Lazarević 2013b).

Nella botanica, i nomi per alcune specie corrispondono in entrambe le lingue, ma a volte ci possiamo accertare che non si tratta di piante uguali soltanto basandoci sulle loro denominazioni latine: olmo bianco (ulmus laevis) non equivale al serbo beli brest (ulmus effusa); biancospino è il nome italiano per i serbi crveni glog e beli glog, ma il primo in italiano è biancospino selvatico (Crataegus laevigata), mentre il secondo è biancospino comune (Crataegus monogyna).

Il serbo beli sport indica il tennis, mentre in italiano il sintagma sport bianco viene usato meno, ma per parlare dello sci.

\subsubsection{NERO}

Alcuni concetti che in serbo sono simboli della morte in italiano hanno un valore differente (crna zastava simboleggia la morte o il funerale, mentre la bandiera nera per un italiano di solito rappresenta il simbolo dell'anarchia, crna misa indica la messa per i morti, a differenza dell'italiano in cui si intende un rituale satanista), ma ci sono anche quelli le cui traduzioni letterali coincidono, ma in ciascuna lingua hanno un significato specifico, caratteristico della civiltà in questione. E il caso quando si tratta di alcune espressioni che, ognuna in proprio modo, illustrano un periodo della storia serba e italiana.

I termini Crna ruka e mano nera si riferiscono a fenomeni differenti, ciascuno specifico della storia serba oppure italiana e italo-americana: il termine serbo è legato alla fine della dinastia degli Obrenović agli inizi del Novecento, dopo la congiura tramata dall'organizazione omonima, mentre 
in italiano mano nera è associata alla storia della mafia e della criminalità organizzata in generale, e in quel contesto può riferirsi a diverse associazioni per delinquere.

\subsection{Falsi amici parziali}

Gli esempi a seguire rappresentano falsi amici che in una lingua ricoprono campo semantico più ampio rispetto all'altra, e quindi grazie alla polisemia dell' espressione polirematica in una lingua può esistere una parziale equivalenza traduttiva, ma soltanto per uno dei possibili significati.

\subsubsection{ROSSO}

Nella letteratura storica, oltre al termine guardia rossa per i rivoluzionari russi, l'italiano possiede anche la forma al plurale - guardie rosse - che si riferisce all'Armata cinese (in serbo kineska Crvena armija), e così rileviamo una molteplice coincidenza tra le collocazioni armata rossa, guardiale rossa/e, crvena garda e Crvena armija.

L'italiano ha più collocazioni legate al sistema bancario che associano il colore rosso ai debiti, cioè a un bilancio passivo, mentre le espressioni del genere sono relativamente recenti nella lingua serba, e pertanto non ancora annoverate dal corpus, e come molte altre neoformazioni vengono marcate con le virgolette (avere il conto in rosso, andare/essere in rosso = biti u minusu, ma di recente anche biti o imati račun "u crvenom"). Ovviamente qui riscontriamo un esempio di equivalenza; ma l'italiano, poi, fa distinzione fra i termini di clausola rossa e clausola verde ${ }^{3}$; nel serbo, il secondo termine non esiste, ma il suo significato rientra in una delle forme di clausola rossa ("crvena" klauzula ), il che potrebbe ancora condurre ad equivoci.

\subsubsection{VERDE}

Ambedue le lingue associano il verde all'esuberanza delle piante e della natura in generale, alla crescita e alla gioventù. Però la gioventù può essere sinonimo dell'immaturità, anche in senso figurato, come nella locuzione serba biti još mlad $i$ zelen, che non va confusa con le espressioni italiane che si riferiscono alla vitalità (vivo e verde, essere nel verde degli anni).

3 "clausola verde: condizione di utilizzazione di crediti contro presentazione di documenti comprovanti l'immagazzinamento, anche parziale, delle merci in attesa di spedizione" (Zingarelli 2008).

4 “clausola rossa: condizione di utilizzazione di crediti documentari, per cui la banca è autorizzata a concedere anticipi a un esportatore, prima della presentazione dei documenti di spedizione" (Zingarelli 2008). 


\subsubsection{GRIGIO}

Bisogna fare distinzione tra la collocazione italiana mercato grigio (calco dall'ingl. grey market) e la serba sivo tržište. Nel serbo, sivo tržište si usa come sinonimo di siva ekonomija (economia grigia), tutti e due i termini legati ai guadagni illeciti e al profitto ottenuto al di fuori del controllo dello stato, e così associati anche ai concetti del mercato nero, borsa nera, lavoro nero (crno tržište, crna berza, rad na crno) ecc. A differenza del serbo, il calco italiano mantiene il significato originale del termine inglese che fa distinzione tra il mercato nero e quello grigio: il mercato grigio non è illecito né viola la normativa vigente, ma trova un modo di aggirare i canali del mercato ufficiale, e di moltiplicare il profitto rispetto alle procedure regolari mediante certe "scorciatoie", evadendo i costi di burocrazia. Comunque, visto che questi significati non sono del tutto dissimili, anzi sono reciprocamente intrecciati, li classifichiamo come falsi amici parziali.

\subsubsection{GIALLO}

La collocazione žuto zlato può avere l'uguale significato all'italiano oro giallo, ma nel serbo può essere usata anche come metafora, parlando del granturco.

\subsubsection{AZZURRO}

Alcuni termini coprono un significato simile ma non del tutto uguale nelle rispettive lingue, e talvolta non si tratta che di una sfumatura di differenza (plava knjiga - libro dalla copertina azzurra in cui il Ministero degli Esteri serbo pubblica documenti di maggiore importanza, libro azzurro - la sua controparte britannica, cioè l'unica differenza è che gli italiani non usano questa collocazione nel contesto nazionale), oppure, oltre a quello comune alle due lingue, l'espressione in una lingua possiede un valore che non caratterizza l'altra; ad esempio, secondo la definizione del dizionario Treccani, nastro azzurro denomina "il nastro che in Italia sostiene la decorazione al valor militare, e il nastrino che si porta in luogo della decorazione stessa (per metonimia, un n. azzurro, la persona stessa insignita della decorazione)". Una complicazione in più è rappresentata dal fatto che pure un marchio popolare della birra italiana si chiama Nastro Azzurro, all'onore del primo transatlantico italiano che ha ricevuto l'omonimo riconoscimento per il primato di velocità nell'attraversare l'Atlantico.

\subsubsection{BIANCO}

In serbo, bela smrt significa morte per assideramento, di solito nella neve profonda. Il significato uguale esiste anche in italiano, ma la locuzione 
stessa con quel valore è relativamente poco usata in confronto alle altre accezioni per le quali il serbo non ha equivalenti. Le locuzioni morte bianca o omicidio bianco spesso indicano un incidente al posto di lavoro con esito mortale, soprattutto nei cantieri edili che trascurano le misure di sicurezza; poi, morte bianca può significare la morte improvvisa dei neonati, chiamata anche "morte in culla" (Lazarević 2013a).

L'italiano notte bianca, oltre al fenomeno nordico, in Italia si può riferire anche alla manifestazione annuale dell'apertura notturna delle istituzioni culturali come musei, gallerie, librerie e così via. La collocazione serba belo zlato, oltre che la sfumatura dell'oro come l'italiano oro bianco, può metaforicamente indicare anche il platino.

Ancora nell'ambito storico, il serbo bela garda (guardia bianca o anche Armata bianca), oltre ai combattenti controrivoluzionari durante la rivoluzione russa, potrebbe denominare anche i gruppi clericofascisti in Slovenia durante la Seconda guerra mondiale, il che raramente rientra tra i valori dell'italiana guardia bianca; d'altronde, il termine guardia bianca nell'italiano può denominare anche una fazione anticomunista finlandese durante la guerra civile russa, un significato non condiviso dal termine serbo.

\subsubsection{NERO}

Nella zoologia a volte insorgono difficoltà di traduzione uguali a quelle già affacciate nel campo della botanica. L'unico modo sicuro di accertarsi dell'equivalenza di un termine è controllare il nome latino di una specie. Così, ad esempio, risulta che il serbo crni orao non si riferisce allo stesso uccello dell'italiano aquila nera: crni orao (Aquila clanga) corrisponde all'italiano aquila anatraia maggiore, mentre aquila nera (Aquila verreauxii) in serbo viene tradotta come afrički crni orao.

\section{CONCLUSIONE}

Tra i sintagmi cromatici, se sono presenti in ambedue le lingue, italiana e serba, in genere riscontriamo un grado di equivalenza traduttiva abbastanza alto. Tali somiglianze, però, non soltanto in questo caso ma per quanto riguarda tutti i colori basici, possono rappresentare anche una fonte di equivoci nei casi in cui gli usi e le terminologie italiana e serba non coincidono, il che tra l'altro conduce alle traduzioni erronee, e specialmente nei termini che sembrano traduzione letterale dell'espressione originale.

Dal punto di vista morfologico, la maggioranza degli esempi consiste dei sintagmi nominali (nome + aggettivo cromatico), e il resto è costituito da quelli verbali (verbo + aggettivo cromatico, oppure verbo + preposizione + agget- 
tivo cromatico / sintagma nominale), che però rappresentano un campione marginale.

Tra le polirematiche relative ai colori, non sono stati rilevati esempi per tutti i colori basici, precisamente per arancione, viola e marrone. Questo è comprensibile se si prende in considerazione il fatto che nemmeno il corpus intero annovera esempi di collocazioni per tutti e undici i colori basici. Quindi possiamo concludere che, se già non si riscontrano esempi di polirematiche equivalenti per alcuni colori, tanto meno li si può trovare per i casi specifici di false analogie.

Le differenze culturali si manifestano più ovviamente negli esempi delle espressioni legate alla storia. Quindi il traduttore deve stare particolarmente attento a non dare per scontato il significato di una polirematica che in apparenza corrisponde letteralmente al sintagma rispettivo nella lingua di destinazione. Bisogna sempre prendere in considerazione il contesto storico e culturale della civiltà cui è riferita una determinata espressione.

Questi e simili esempi illustrano come le differenze e apparenti somiglianze nelle locuzioni e collocazioni cromatiche possano servire da testimonianza di relazioni non solo tra le lingue, ma anche tra due culture e civiltà. Certe espressioni cromatiche sono caratteristiche di una sola lingua e cultura, mentre nell'altra richiedono spiegazioni aggiuntive oppure una traduzione parafrasata, perfino in alcuni casi in cui possono sembrare semplici calchi.

\section{BIBLIOGRAFIA}

Benedik, A. \& Gabrovšek, D. (2004). False friends: slovensko-angleški slovar lažnih prijateljev. Ljubljana: Cankarjeva založba.

Benincà, P. \& Penello, N. (2006). Alcune considerazioni sui "faux amis". In E. Cresti (a cura di), Prospettive nello studio del lessico italiano (Vol. II, pp. 607-614). Firenze: FUP.

Berlin, B. \& Kay, P. (1999). Basic Color Terms: Their Universality and Evolution. Stanford: CSLI Publications.

Buntić, D. (1990). Morfološko-semantički "lažni prijatelji” u obostranim francusko-srpskohrvatskim i hrvatskosrpsko-francuskim rečnicima. Strani jezici, 3, 106-111.

Ivir, V. (1984). Teorija i tehnika prevođenja. Sremski Karlovci: Centar Karlovačka gimnazija / Novi Sad: Zavod za izdavanje udžbenika.

Klajn. I. (1971). Uticaji engleskog jezika u italijanskom. Beograd: Filološki fakultet Beogradskog univerziteta. 
Kudela, J. (1981). Skica jedne tipologije "lažnih prijatelja" francuskog porekla u srpskohrvatskom jeziku. Naučni sastanak slavista u Vukove dane, 7 , 233-250.

Lazarević, R. (2013a). Leksičko-semantičko polje boja u italijanskom i srpskom jeziku. (Dottorato di ricerca, non pubblicato; Beograd: Filološki fakultet).

Lazarević, R. (2013b). La traduzione dei termini cromatici dell'italiano in serbo e viceversa. Italica belgradensia, 1, 227-234.

Lewis, K. (2008). Dva aspekta neodređenosti pojma "lažni prijatelji”. In M. Prihoda \& H. Vankova (a cura di), Slavistika dnes. Vlivy a kontexty (pp. 173-189). Červeny Kostelec/Praha: P. Mervart/Univerzita Karlova, Filosofická fakulta.

Matešić, J. (1995). Prividnost katkad vara. O "faux amis" frazeologiji. Filologija, 24-25, 239-245.

Vuletić, J. (2010). Lažni prijatelji u lingvistici, njihova klasifikacija i pokušaj osvrta na jezički par nemačko-srpski. Nasleđe, 15/2, vol. 7, 183-198.

\author{
FALSE FRIENDS OF EVERY COLOUR: SEMANTIC TRAPS \\ IN THE TRANSLATION OF ITALIAN AND SERBIAN EXPRESSIONS \\ CONTAINING BASIC COLOUR TERMS
}

\begin{abstract}
Summary
The article treats Italian and Serbian syntagmatic expressions containing adjectives for basic colour terms (as listed in Berlin and Kay's theory of 1969), including both idioms and fixed collocations. An expression can cover more different meanings in one language than in another (morte bianca, nastro azzurro, žuto zlato), or the meanings of two apparently literally equivalent translations can differ completely (peste bianca/bela kuga, valuta verdelzelena valuta). Such examples of phrases without real semantic equivalence can be found in the most various spheres, which makes it necessary for a translator to carefully verify possible meanings of the phrases that at first sight may appear literally equivalent. This article brings illustrative examples of so-called "false friends" among the syntagmatic expressions containing basic colour terms which could pose difficulties in translation from Italian to Serbian and vice versa.
\end{abstract}

Keywords: basic colour terms, false friends, Italian, semantics, Serbian, translation 
